\title{
Riesgo ambiental y conservación preventiva en colecciones paleontológicas: un abordaje incipiente en la República Argentina
}

Para citar este artículo: Susana Mariel Devincenzi (2021). Riesgo ambiental y conservación preventiva en colecciones paleontológicas: un abordaje incipiente en la República Argentina. Publicación Electrónica de la Asociación Paleontológica Argentina 21 (1): 107-117.

Link a este artículo: http://dx.doi.org/10.5710/PEAPA.05.12.2020.341

(C)2021 Devincenzi

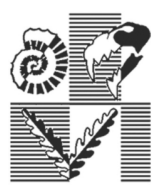

ISSN 2469-0228

\section{Asociación Paleontológica Argentina}

Maipú $6451^{\circ}$ piso, C1006ACG, Buenos Aires

República Argentina

Tel/Fax (54-11) 4326-7563

Web: www.apaleontologica.org.ar
Acceso Abierto

Open Access

This work is licensed under

CC BY-NC 4.0

(c) (1) (8) 


\title{
RIESGO AMBIENTAL YCONSERVACIÓN PREVENTIVA EN COLECCIONES PALEONTOLÓGICAS: UN ABORDAJE INCIPIENTE EN LA REPÚBLICA ARGENTINA
}

\author{
SUSANA MARIEL DEVINCENZI
}

Instituto Argentino de Nivología, Glaciología y Ciencias Ambientales (IANIGLA), CCT-CONICET-Mendoza. Av. Ruiz Leal s/n, Parque General San Martín, M5500 Mendoza, Argentina.sdevincenzi@mendoza-conicet.gob.ar,devincenzisusana@gmail.com

SMD: https://orcid.org/0000-0002-4716-1309

Resumen. La conservación preventiva de los bienes patrimoniales estuvo inicialmente asociada a las variables ambientales de los museos que los custodiaban. Las colecciones paleontológicas, si bien menos susceptibles que otras colecciones biológicas, no son ajenas a esta problemática; sin embargo, la comunidad científica ha sido muchas veces reticente a esta realidad. A partir de la década de 1970, diversas instituciones del Reino Unido, Estados Unidos de América, Canadá y algunos países europeos dieron impulso a esta temática; publicaron numerosas contribuciones que trataron en detalle las causas y las formas de mitigación de los daños potenciales a los ejemplares fósiles y, en años recientes, incluyeron técnicas espectroscópicas (no invasivas) a casos de estudio. Este trabajo tiene como objetivo sintetizar el conocimiento de los factores ambientales que representan un riesgo para las colecciones paleontológicas, una temática cuyo abordaje, como parte de la conservación preventiva, es incipiente en nuestro país.

Palabras clave. Riesgo ambiental. Conservación preventiva. Paleontología. Argentina.

Abstract. ENVIRONMENTAL RISK AND PREVENTIVE CONSERVATION IN PALEONTOLOGICAL COLLECTIONS: AN INCOMING APPROACH IN ARGENTINA. The preventive conservation of heritage assets was initially associated with the environmental variables of the museums in which they are stored. Paleontological collections, although less susceptible than other biological collections, are no stranger to this problem. However, the scientific community has often been reluctant to this reality. At the beginning of the 1970s, institutions of the United Kingdom, the United States of America, Canada, and some European countries provided the impulse to this issue with the publication of numerous contributions that discussed in detail the causes and mitigating methods of potential damage to fossils; recently, they included non-invasive spectrometric techniques to case studies. This paper aims to synthesize the knowledge of the environmental factors that represent a risk for paleontological collections, a subject whose approach is incipient in our country as part of preventive conservation.

Keywords. Environmental risk. Preventive conservation. Paleontology. Argentina.

EL INTERÉS por conservar el patrimonio histórico y artístico está íntimamente asociado al concepto de la conservación preventiva. Este vocablo, acuñado desde la década de 1970 y que en la actualidad se refiere a todas aquellas medidas o acciones que pueden evitar o prevenir el deterioro del patrimonio (de Guichen, 1999), en sus inicios se asoció al control del microclima de los museos que albergaban bienes patrimoniales; ya que el uso de la calefacción a carbón, las lámparas de gas y los contaminantes generados por la Revolución Industrial habían producido problemas antes inexistentes (García Fernández, 2013). Hacia la década de 1930, el Dr. H. Plenderleith, una figura de relevancia mundial en el campo de la conservación durante mediados del siglo
XX y miembro del British Museum en Londres (Inglaterra), junto a J. A. Macintyre analizaron la descamación de las diferentes capas en pinturas de caballete y aconsejaron eliminar la variación diaria y estacional de la humedad a las que estaban sometidas; reportaron sus investigaciones en un folleto titulado "Some Notes on Atmospheric Humidity in Relation to Works of Art" (Algunas notas sobre la humedad atmosférica en relación con obras de arte) que fue publicado por el Courtauld Institute of Art de la Universidad de Londres (Oddy, 2011). Fue así como el daño causado en obras de arte por efecto de la temperatura, la humedad, la luz y el polvo atmosférico se convirtió en motivo de análisis para los curadores de colecciones de arte desde principios del siglo XX. 
Los fósiles son menos vulnerables al deterioro que otros materiales biológicos, pero no fueron ajenos a esta problemática, aunque la concientización de la comunidad paleontológica fue posterior y muchas veces inexistente al día de hoy. Durante años, las colecciones de invertebrados fósiles habían sido atendidas por los investigadores que las utilizaban para sus estudios y recién desde la década de 1970, con la profesionalización de la gestión y el advenimiento del collection manager, tuvieron una mejora en su administración y preservación (White, 2000). A partir de 1974, las acciones llevadas a cabo por el Geological Curators' Group (Inglaterra), no fueron indiferentes a la necesidad de la labor curatorial del material geológico. Con la colaboración de la Palaeontological Association (Inglaterra), se promovieron encuentros sobre el manejo y el cuidado de las colecciones paleontológicas, a los que asistieron fundamentalmente curadores británicos, pero también delegados de Estados Unidos de América, Canadá, Noruega, Francia y Alemania Occidental (Bassett, 1979). Sin embargo, años más tarde, Howie (1978), miembro del Departamento de Paleontología del British Museum (Natural History) en Londres (Inglaterra) (BM(NH))—el cual a partir de 1992 pasó a llamarse Natural History Museum (NHM) — planteó que los curadores de colecciones de historia natural no eran totalmente conscientes de que los mismos factores ambientales que dañaban objetos de arte podían deteriorar algunos minerales y fósiles. Por su parte, Shute y Cleal (1987) subrayaron la falta de interés de la comunidad científica que difícilmente financiaba este tipo de abordaje. Años más tarde, Waddington (2000) manifestó que esta problemática era subestimada aún por algunos paleontólogos.

En ese contexto, diversos autores analizaron el deterioro causado a las colecciones paleontológicas por variables ambientales y las medidas de mitigación para lograr su conservación preventiva. El objetivo de este trabajo es resumir la información sobre los factores ambientales que representan un riesgo para las colecciones paleontológicas, un tema poco abordado en nuestro país.

\section{MATERIALES Y MÉTODOS}

Se realizó una revisión bibliográfica de los factores ambientales que pueden dañar las colecciones paleontológicas, que se considera representativa de la literatura más relevante, si bien no se pudieron consultar ciertas publicaciones de acceso restringido.

Se tuvo acceso virtual a muchas publicaciones utilizando palabras clave como fósil, paleontología, conservación preventiva, paleontological collections, care and management, microenvironment storage o damage, entre otras. Solo se utilizaron estudios realizados en instituciones tales como museos, universidades, organismos gubernamentales y organizaciones no gubernamentales. En los trabajos revisados, se incluyen artículos publicados en revistas, capítulos de libros y ponencias presentadas en reuniones científicas. El análisis cuantitativo de las fuentes primarias permitió examinar la distribución geográfica, cronológica y de formato de la bibliografía consultada y, a partir de las fuentes secundarias (entendiendo como tales las citas bibliográficas de los trabajos revisados), se comprobaron cuáles fueron los autores más citados.

\section{RESULTADOS}

La lectura de la vasta bibliografía recopilada indica que, al igual que en las obras de arte, en el ámbito de la paleontología los factores ambientales fueron considerados variables para alcanzar la conservación preventiva. La humedad relativa, la temperatura, la luz, el polvo atmosférico y los agentes biológicos se analizaron como elementos potenciales de deterioro de una unidad de colección (ejemplares e información asociada), siendo la humedad uno de los temas que ha despertado mayor interés en los curadores.

\section{Humedad relativa}

Se entiende por humedad relativa (HR) la relación entre la cantidad de vapor de agua contenida en el aire y la máxima cantidad que puede albergar a una temperatura dada. Se mide en valores porcentuales y está íntimamente relacionada con los cambios en la temperatura. El aumento de la temperatura implica una mayor cantidad de vapor de agua para saturar un determinado volumen y, por lo tanto, una reducción de la HR (Crespo y Viñas, 1884).

La humedad es un factor que afecta diferentes tipos de ejemplares fósiles, lo cual se debe no solo a la naturaleza del ejemplar, sino también a las características de la roca sedimentaria asociada.

Uno de los tipos de fósiles más vulnerable es el que está 
piritizado. La pirita $\left(\mathrm{FeS}_{2}\right)$ es un mineral perteneciente a la clase de los sulfuros que cristaliza en el sistema isométrico, su color es amarillo pálido y su brillo es metálico. Posee un polimorfo, la marcasita, que cristaliza en el sistema ortorrómbico. El FeS 2 se presenta en algunos restos fósiles debido al reemplazo de la materia orgánica en un medio reductor durante el proceso de fosilización. El Mal de la Pirita (Pyrite Desease o Pyrite Decay) es uno de los problemas más importantes observados en las colecciones paleontológicas, siendo común también en colecciones mineralógicas y arqueológicas. Se origina a partir de la oxidación del sulfuro de hierro, con la subsiguiente formación de sulfatos hidratados y de dióxido de azufre que, en presencia de humedad, favorecen la formación de ácido sulfúrico $\left(\mathrm{SO}_{4} \mathrm{H}_{2}\right)$ (Buttler, 1994a). Su efecto en el fósil es el agrietamiento del ejemplar y la presencia de eflorescencias blancas o amarillentas; cuando el daño es avanzado, se caracteriza por la rotura parcial o total del ejemplar, e incluso de la etiqueta que lo acompaña (Fig. 1.1-2). Bather (1908), miembro del BM (NH), informó que el mayor problema para la preservación de los fósiles era su asociación con la pirita y la marcasita. Howie (1978, 1979a, 1979b) y Buttler (1994a) abordaron las causas, el tratamiento y las formas de prevención ante un proceso que es irreversible una vez iniciado. Newman (1998) resumió los diferentes mecanismos (molecular, bacteriológico y electroquímico) propuestos para que se produzca la oxidación de la pirita y el rol que cumplen en la reacción no solo la humedad, sino otros factores como la temperatura, la concentración de oxígeno, el pH y el tamaño de los cristales de pirita. A lo largo del siglo XX, se propusieron diferentes tratamientos para aislar los ejemplares de la atmósfera. Buttler (1994a) mencionó el uso de revestimientos como laca (shellac), baquelita y acetato de polivinilo (PVA), pero sin los resultados esperados. Cornish y Doyle (1984) y Cornish (1986) reportaron el uso del tioglicolato de ethanolamina. Se propuso también el uso de vapores de amonio para la remoción y la neutralización de los productos de oxidación (Buttler, 1994a y bibliografía allí incluida). Las variables que aceleran la oxidación y el deterioro de los especímenes y los tratamientos sugeridos fueron revisados por Montero y Diéguez (2001), McDonald et al. (2005), Shinya y Bergwall (2007) y Larkin (2011), entre otros. Baeza Chico y Menéndez (2005), en el Museo Geominero en Madrid
(España), y Holmberg (2000), en el Naturhistoriska riksmuseet en Estocolmo (Suecia), analizaron el deterioro de fósiles piritizados y su relación con las características del agregado cristalino de la pirita y con el tipo y contenido de las arcillas asociadas. Doyle (2003), con el objetivo de resguardar ejemplares de Lepidostrobus fimbriatus con presencia de decaimiento de pirita en las colecciones paleontológicas del NHM, aplicó la microclimatización de gabinetes con el uso de Marvelseal ${ }^{\circledR} 470$ (film de polietileno, aluminio y polipropileno resistente al paso de humedad y a la ruptura) y de Art-Sorb ${ }^{\circledR}$ (agente de control de humedad) acondicionado a valores promedio de $45 \%$ de $\mathrm{HR}$, un poco más alto de lo recomendable, debido a que la asociación del fósil con lutitas podría ocasionar el agrietamiento del ejemplar.

Rouchon et al. (2012), en el Muséum national d'Histoire naturelle en París (Francia), analizaron y categorizaron el daño ocasionado en plantas fósiles con presencia de sulfuro de hierro y utilizaron la espectroscopia Infrarroja con Transformada de Fourier (FTIR) y la espectroscopia Raman para la identificación de sulfatos en eflorescencias. Baars y Horak (2018) resaltaron la importancia de la utilización de estos métodos no invasivos, que permiten determinar las fuerzas de enlace y la coordinación entre átomos, la dinámica de la red y la presencia de poliedros en la materia, para detectar los cambios físicos y químicos originados en ejemplares piritizados susceptibles a la oxidación e hidratación, y destacaron además la necesidad de contar con protocolos para monitorearlos de manera rutinaria. Becherini et al. (2018) reportaron el deterioro por decaimiento de pirita causado en Latanites sp., perteneciente a una colección histórica de plantas fósiles en el Museo di Geologia e Paleontologia en Padova (Italia), estudiaron el producto de su degradación con difractómetro de rayos X y espectroscopia Raman y analizaron el monitoreo de la microclimatización de los ejemplares en vitrinas y salas. Cornish (1986) y Stooshnov y Buttler (2001) describieron técnicas utilizadas en el campo de la conservación para tratar tarjetas históricas dañadas debido a su almacenaje junto a ejemplares que fueron atacados por el decaimiento de la pirita. Hodgkinson y Martin (2004) y Del Favero et al. (2012) presentaron otros casos de estudios sobre la degradación, el monitoreo y la restauración de fósiles piritizados. Odin et al. (2013) reprodujeron en laboratorio los daños ocasionados en fósiles con sulfuros de hierro 
con el objetivo de estudiar sus características. En 2015, el NHM inició un proyecto conocido como "Airless Project" para combatir el deterioro de la pirita mediante microambientes con bajo contenido de oxígeno (Allington-Jones y Trafford, 2017). Si bien se han propuesto diferentes tratamientos para evitar el deterioro de los ejemplares piritizados, actualmente el control medioambiental sería la medida más apropiada.

Los subfósiles son también susceptibles a valores inapropiados de HR. Se entiende como tal aquellos restos pleistocenos que sufrieron una fosilización parcial y que contienen restos de colágeno (e.g., dientes, cuernos, marfil, cuerpos congelados en condiciones excepcionales de permafrost). En condiciones de HR alta, los subfósiles tienden a aumentar su volumen y aparecen eflorescencias; por el contrario, las condiciones de baja HR pueden causar contracción y agrietamiento (Buttler, 1994b). También son sensibles a cambios ligeros de HR y presentan su peor escenario ante un cambio del 10\% de HR en un día (Andrew, 1996). McDonald et al. (2005) recomendó valores entre 45$55 \%$ de HR para el almacenaje. Doyle (1983) utilizó una emulsión de acetato de polivinilo para la preservación de los subfósiles en ambientes extremadamente secos para evitar la fragilidad del ejemplar. Barrón-Ortiz et al. (2018) presentaron el caso de vertebrados saturados en agua recuperados de un ambiente lacustre (Cold Lake, Canadá) del Cuaternario tardío; probaron distintos métodos de secado para su mejor preservación, concluyendo que los mejores resultados se obtuvieron mediante el secado controlado al aire en primer término, seguido del secado por solvente. En contraste, la aplicación de liofilización al vacío originó agrietamiento y delaminación de los ejemplares. Sin embargo, alertaron de que el uso de solvente podría alterar futuros estudios moleculares y químicos (e.g., extracción de ADN antiguo, análisis de isótopos estables, análisis de elementos traza).

El ámbar, resina fósil, también puede deteriorarse por factores ambientales, siendo el oxígeno atmosférico el principal factor de su degradación (Corral, 1999 y bibliografía allí incluida). Las características de su deterioro son el agrietamiento superficial, el material pulverulento y la pérdida de brillo. Corral (1999) detalló los valores orientativos de HR para la conservación del ámbar, siguiendo las sugerencias de diferentes autores. Bisulca et al. (2012) analizaron la degradación en algunas muestras de ámbar del CretácicoHoloceno de la colección de la División de Zoología de Invertebrados del American Museum of Natural History (AMNH) en Nueva York (Estados Unidos de América) y recomendaron la necesidad de mantener valores de HR entre $35-45 \%$.

La humedad es también un factor de riesgo para los ejemplares paleobotánicos. El problema principal es la exfoliación de las cutículas carbonosas de la matriz, debido al secado diferencial, proceso que una vez que comienza se torna irreversible, con la pérdida del material (Fig. 1.3). CoIlinson (1986) recomendó secar lentamente el espécimen colectado, evitando las fluctuaciones de HR y la caída por debajo del $50 \%$ en áreas de almacenaje; además, propuso evitar el uso de materiales con propiedades electrostáticas, como el acetato de celulosa, en los contenedores.

Los coprolitos, extraídos a menudo de ambientes áridos, deben almacenarse en ambientes con baja HR, envueltos en bolsas de polietileno y con desecantes pre-acondicionados (Shelton, 1994).

Para las colecciones formadas por preparaciones microscópicas cuyos medios de montaje son preferentemente solubles en agua, se advierte la formación de hongos cuando se encuentran en ambientes húmedos; en contraste, se produce la decoloración y el agrietamiento del medio de montaje en ambientes secos (Museums and Galleries Commission, 1992).

El papel de las tarjetas puede dañarse potencialmente por un exceso de humedad que causa su descomposición por hidrólisis de la celulosa y reblandece los aprestos utilizados en su fabricación; por otro lado, un ambiente seco propiciará su fragilidad, debido a la pérdida de puentes interfibrilares de la celulosa. Los valores altos de HR (y de temperatura) favorecen la presencia de hongos y de insectos sobre el papel (Crespo y Viñas, 1984). Recientemente, Rojas et al. (2020) analizaron el impacto que los cambios de temperatura y HR tuvieron sobre la Colección Paleontológica de la Universidad de la República en Montevideo (Uruguay). Estos autores concluyeron que el mayor riesgo proviene de las fluctuaciones de HR y que los contenedores amortiguan esta variable de manera diferente, sugiriendo que aquellos de plástico y cerrados son más efectivos que los de cartón común. 
Los valores de HR recomendados para los diferentes tipos de fósiles, soportes y tarjetas que los acompañan son ligeramente disímiles según los autores consultados (Tab. 1) y deben entenderse como estimativos, pero hay una coincidencia en la necesidad de evitar fluctuaciones de humedad. Además, es importante tener en cuenta que una tasa de cambio repentino de la HR fuera de los contenedores de los ejemplares se refleja 24 horas después en su interior (Simmons y Muñoz-Saba, 2005).

\section{Temperatura}

Para toda colección de historia natural, se considera, en general, que las fluctuaciones térmicas generan deterioro y que las temperaturas altas aceleran las reacciones químicas y la proliferación del ataque biológico. Ante cambios abruptos de temperatura (thermal shock), los dientes de vertebrados fósiles pueden presentar fragilidad y descamación del esmalte (Corral, 2012). El rango de temperatura recomendado para el almacenaje de ejemplares piritizados o con marcasita está entre $16-22{ }^{\circ} \mathrm{C}$, con valores de HR de 20-

TABLA 1 - Recomendaciones sobre valores de HR según distintos autores

\begin{tabular}{|c|c|c|}
\hline Material almacenado & Autores & Consideraciones sobre valores de HR \\
\hline \multirow[t]{8}{*}{ Ejemplar piritizado } & Howie (1978) & Recomendado $\leq 50 \%\left(\mathrm{a} 25^{\circ} \mathrm{C}\right)$ \\
\hline & Howie (1979a) & El deterioro se inicia $55-60 \%$ \\
\hline & Howie (1979b) & Pocos días con valores de $60 \%$ pueden iniciar el deterioro \\
\hline & Buttler (1994a) & Recomendado $<50 \%$ \\
\hline & National Park Service (1998) & El daño puede desencadenarse a partir de $60 \%$ \\
\hline & Doyle (2003) & Recomendado 50\% si está asociado con lutitas \\
\hline & Museums, Libraries and Archives Council (2004) & Recomendado $20-30 \%$ \\
\hline & McDonald et al. (2005) & Recomendado $\leq 45 \%$. Si el deterioro comenzó, reducir $\mathrm{a} \leq 30 \%$ \\
\hline \multirow[t]{4}{*}{ Subfósiles } & Howie (1978) & Recomendado $45-60 \%$ \\
\hline & Andrew (1996) & $\begin{array}{l}\text { Colecciones antiguas: recomendado } 50-60 \% \text {; evitar valores } \\
\text { menores a } 40 \%\end{array}$ \\
\hline & Museums, Libraries and Archives Council ( 2004) & Recomendado 50\% (no menor al 40\%) \\
\hline & McDonald et al. (2005) & Recomendado $45-55 \%$ \\
\hline \multirow[t]{5}{*}{ Ámbar } & Shelton (1994) & Recomendado 55\% \\
\hline & Corral (1999) y bibliografía alli incluida & Recomendado $35-45 \%$ \\
\hline & Corral (1999) y bibliografía allí incluida & Recomendado $50-60 \%$ \\
\hline & Corral (1999) y bibliografía alli incluida & Recomendado $45-55 \%$ \\
\hline & Bisulca et al. (2012) & Recomendado $35-45 \%$ \\
\hline $\begin{array}{l}\text { Fósiles asociados a } \\
\text { matriz arcillosa }\end{array}$ & Museums, Libraries and Archives Council (2004) & Se pueden desintegrar por debajo del $40 \%$ \\
\hline Cutículas carbonosas & Collinson (1986) & Evitar caídas por debajo del 50\% \\
\hline Papel & Museums and Galleries Commission (1992) & Recomendado $55-65 \%$ \\
\hline \multirow[t]{2}{*}{$\begin{array}{l}\text { Preparaciones } \\
\text { microscópicas }\end{array}$} & Museums and Galleries Commission (1992) & Recomendado $60 \%$ \\
\hline & Neuhaus et al. (2017) & Recomendado $60 \%$ \\
\hline
\end{tabular}


$30 \%$, al igual que para los subfósiles y ejemplares asociados a matrices arcillosas y pizarras, pero con una HR no inferior al 40\% (Museums, Libraries and Archives Council, 2004). Según algunos autores (Newman, 1998 y bibliografía allí incluida), la tasa de oxidación de la pirita podría duplicarse cada $10^{\circ} \mathrm{C}$ de aumento de la temperatura, a una HR constante. El ámbar también se deteriora por la temperatura. Grimaldi (1993) comunicó el pobre mantenimiento de una colección de ámbar del Báltico en el AMNH, cuyos ejemplares estuvieron almacenados en recintos sin aire acondicionado y con temperaturas entre $90-95^{\circ} \mathrm{F}\left(32,222-35^{\circ} \mathrm{C}\right)$ durante épocas de verano, lo que originó un oscurecimiento del ámbar que dificultaba la observación de las inclusiones. La temperatura recomendada debe estar en un rango entre 16-20 ${ }^{\circ} \mathrm{C}$ y minimizando las fluctuaciones (Corral, 1999 y bibliografía allí incluida). Bisulca et al. (2012) asumieron como beneficioso mantener el ámbar a un valor de temperatura igual o menor a $21^{\circ} \mathrm{C}$. Neuhaus et al. (2017), luego de 25 años de experiencia en las colecciones del Smithsonian Institution en Washington DC (Estados Unidos de América), publicaron una contribución exhaustiva sobre las condiciones de manejo de colecciones biológicas formadas por preparaciones microscópicas, recomendando evitar fluctuaciones de temperatura que podrían producir el agrietamiento de los soportes, debido a los diferentes coeficientes de dilatación del vidrio y del medio de montaje, y recomendaron evitar valores menores a $18{ }^{\circ} \mathrm{C}$. La temperatura recomendada para el almacenaje de papel por el Museums, Libraries and Archives Council (2004) está en un rango entre $13-18^{\circ} \mathrm{C}$.

\section{Luz}

Entre los daños ocasionados en las colecciones paleontológicas por la luz están la fragilidad de los papeles, la decoloración de las tintas usadas en las tarjetas y la fragilidad y oscurecimiento de los adhesivos utilizados en la reparación de ejemplares (Waddington, 2000). Marcos-Fernández et al. (2018) reportaron el análisis colorimétrico realizado sobre un chevron de saurópodo, concluyendo que las variaciones cromáticas producidas por la radiación ultravioleta (UV) obedecieron al grosor de la matriz que cubría al ejemplar. En su contribución, además, presentaron el envejecimiento de las diferentes resinas artificiales utilizadas en conservación-restauración por acción de los rayos UV según distintos autores. Otras investigaciones (Corral, 1999 y bibliografía allí incluida) indicaron que, si bien la luz visible parece no afectar al ámbar, los rayos UV asociados causan su oxidación fotoquímica que es detectada por el análisis espectroscópico FTIR, por lo que recomendaron almacenarlo en oscuridad y utilizar filtros UV durante su exhibición. Pastorelli et al. (2011) indujeron el envejecimiento artificial de muestras de ámbar del Báltico a fin de demostrar el deterioro causado por su interacción con la luz de día. Además, mediante el uso de técnicas analíticas no destructivas (e.g., espectroscopias de Reflectancia Total Atenuada (ATR) y FTIR) confirmaron cambios en el color y en las propiedades químicas de los ejemplares, si bien no quedaba claro si solo la luz visible o también los rayos UV causaban el deterioro. La exposición a los rayos UV del espectro lumínico solar induce, por un lado, al desvanecimiento de las tintas y a la oxidación de los papeles que contienen lignina, causando su amarilleamiento (Crespo y Viñas, 1984) y, por otro, al deterioro de los fósiles que aún retienen material orgánico (McDonald et al., 2005). La luz también puede deteriorar los moldes de goma de látex (National Park Service, 1998).

\section{Polvo atmosférico}

El polvo atmosférico, formado por gases contaminantes y partículas de diferente tamaño y dureza, actúa como un abrasivo y es difícil de eliminar, fundamentalmente de fósi-

Figura 1. Daños ocasionados en colecciones paleontológicas por factores ambientales. Ejemplar de Perisphinctes mairei, MNCNI-40361 y su tarjeta identificatoria de la Colección de Invertebrados Fósiles del Museo Nacional de Ciencias Naturales en Madrid (España), deteriorado por el Mal de la Pirita. Oxfordiano inferior, Villers (Doubs, Francia). 2, Ejemplar de Maltoniceras sp., MGM-719X de la Colección del Museo Geominero en Madrid (España), deteriorado por el Mal de la pirita e intervenido. Jurásico Superior, Formación Oxford Clay (Reino Unido). 3, Exfoliación de partícula carbonosa en una planta fósil (IANIGLA-PD-62) de la Colección Didáctica de Paleontología del IANIGLA CONICET Mendoza, (Argentina). 4, Partículas de polvo atmosférico sobre cutícula de Johnstonia coriacea CT342-IANIGLA vistas bajo el Microscopio Electrónico de Barrido. Triásico

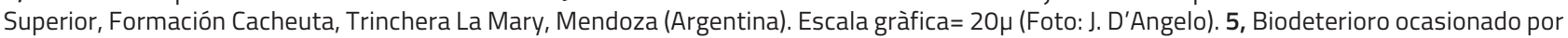
Anobium punctatum en la Colección de Paleontología de Vertebrados del Museo de Ciencias Naturales "Bernardino Rivadavia", Ciudad Autónoma de Buenos Aires (Argentina). Obsérvense las galerías dentro del objeto (Foto: S.M. Álvarez). 6, Tarjeta dañada por microorganismos. 

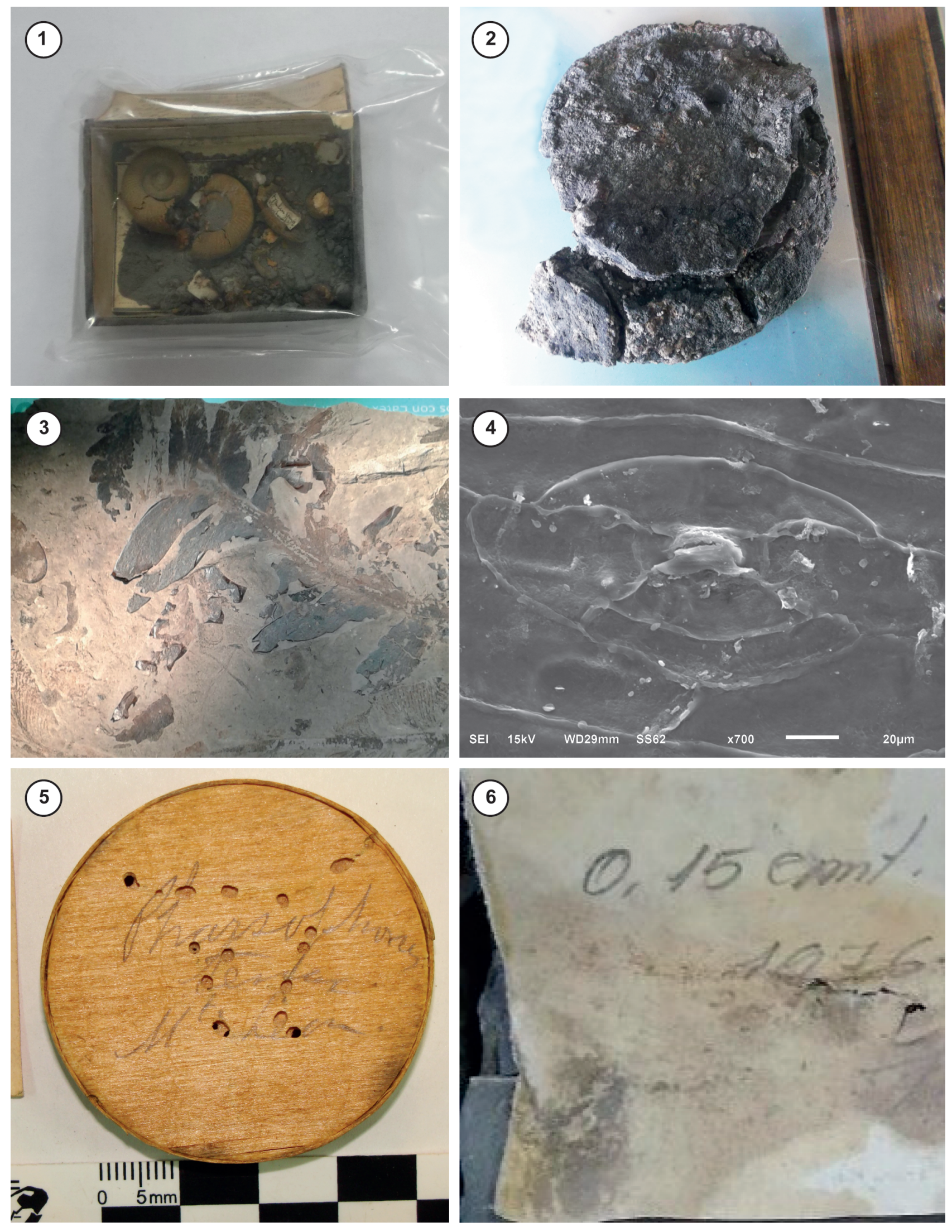
les como graptolites, ejemplares porosos como cnidarios y poríferos o fósiles con superficies rugosas; de igual manera, las cutículas de plantas fósiles pueden desprenderse al tratar de limpiarlas. En la década de 1970, en el BM (NH), se utilizó un lodo compuesto por sepiolita y un filosilicato con propiedades absorbentes, junto con agua e isopropanol o acetona, para limpiar ejemplares no susceptibles a la humedad y a los solventes (Howie, 1979b). El estudio de ejemplares en el microscopio electrónico de barrido puede resultar obstaculizado por la presencia de partículas de polvo que enmascaren estructuras útiles para la clasificación taxonómica (Fig. 1.4). Así mismo, uno de los requisitos básicos para una buena observación microscópica es que tanto las lentes como el montaje de las preparaciones microscópicas estén libres de polvo. No se debe usar agua para limpiar fósiles piritizados, subfósiles o aquellos asociados a matrices arcillosas (Howie, 1979b).

Por todo lo anteriormente expuesto, la autora de este trabajo recomienda almacenar los ejemplares en contenedores cerrados para evitar la contaminación con polvo atmosférico.

\section{Agentes biológicos}

Buttler (1994b) mencionó la posibilidad de crecimiento de moho y hongos en los subfósiles con valores de HR por encima del 70\%. Asimismo, en colecciones paleontológicas se ha observado el ataque de Anobium punctatum, un coleóptero de la familia Anobiidae, en soportes de madera (S. M. Álvarez, com. pers.) (Fig. 1.5) y de microorganismos en tarjetas de papel (Fig. 1.6). Los adhesivos de origen animal son susceptibles de ser atacados por insectos y hongos (Elder et al., 1997).

Sterflinger (2010) investigó exhaustivamente sobre el rol de los hongos en el deterioro del patrimonio cultural, mencionando el daño que pueden ocasionar sobre el papel (e.g., tarjetas de papel que acompañan a los ejemplares), debido fundamentalmente a que excretan celulasa, una enzima que descompone la celulosa. Según el autor, un 55\% de HR representa un valor límite para el crecimiento de hongos, por lo cual el control ambiental debe ajustarse por debajo de este nivel. Informó, además, que la biodiversidad de los hongos en museos y áreas de almacenamiento es acotada (Eurotium sp., Aspergillus sp. y Wallemia sp.) y aumenta cuando la humedad supera el $70 \%$ por períodos pro- longados de tiempo; recomendó el control medioambiental y la limpieza de los lugares de almacenamiento. En los últimos años, el AMNH ha recomendado elaborar un Manejo Integral de Plagas para evitar la presencia de insectos y hongos en las colecciones y no utilizar productos tóxicos por razones de seguridad (http://collections.paleo.amnh.org/ 8/integrated-pest-management).

\section{Análisis de la bibliografía}

La lectura de la bibliografía mencionada indica que, desde la década de 1970 a la actualidad, los factores ambientales que representan un riesgo para las colecciones paleontológicas fueron un tema de consideración, especialmente, en museos y organismos no gubernamentales del Reino Unido (entre otros, NHM, University of Newcastle, National Museum Cardiff, Natural History Ludlow Museum y Museum, Libraries and Galleries), seguidos por instituciones de Estados Unidos de América (entre otros, AMNH, Peabody Museum of Natural History, Museum Sciences of Texas y National Park Service), de países de Europa y de Canadá, cuyas instituciones a partir de la década de 2010 aplicaron técnicas espectroscópicas (no invasivas) a sus casos de estudio (Fig. 2.1-2). La importancia que la comunidad de curadores del Reino Unido le dio a esta temática se confirmó cuando se analizaron las referencias bibliográficas de cada una de las contribuciones revisadas a fin de determinar los autores más citados (Fig. 2.3). El formato de estas contribuciones está representado fundamentalmente por artículos y capítulos de libros. Una de las revistas que se destacan en la publicación de estos trabajos es Geological Curator, perteneciente al Geological Curators' Group, dedicado al mejor cuidado, mantenimiento y uso de colecciones y afiliado a la Geological Society of London (Inglaterra). En cuanto a trabajos publicados en la Argentina referidos a los factores ambientales anteriormente mencionados, se encontraron algunos resúmenes recientes (Fig. 2.4), con menciones a prácticas de conservación preventiva aplicadas en los repositorios respectivos (e.g., Álvarez, 2012; Druetta et al., 2017; Luna et al., 2019) y un resumen referido al caso del holotipo de Panamericansaurus schroederi, un húmero que presentaba un desprendimiento de su capa externa debido a la humedad residual procedente de su ambiente de colecta (Garat y Milani, 2011). 

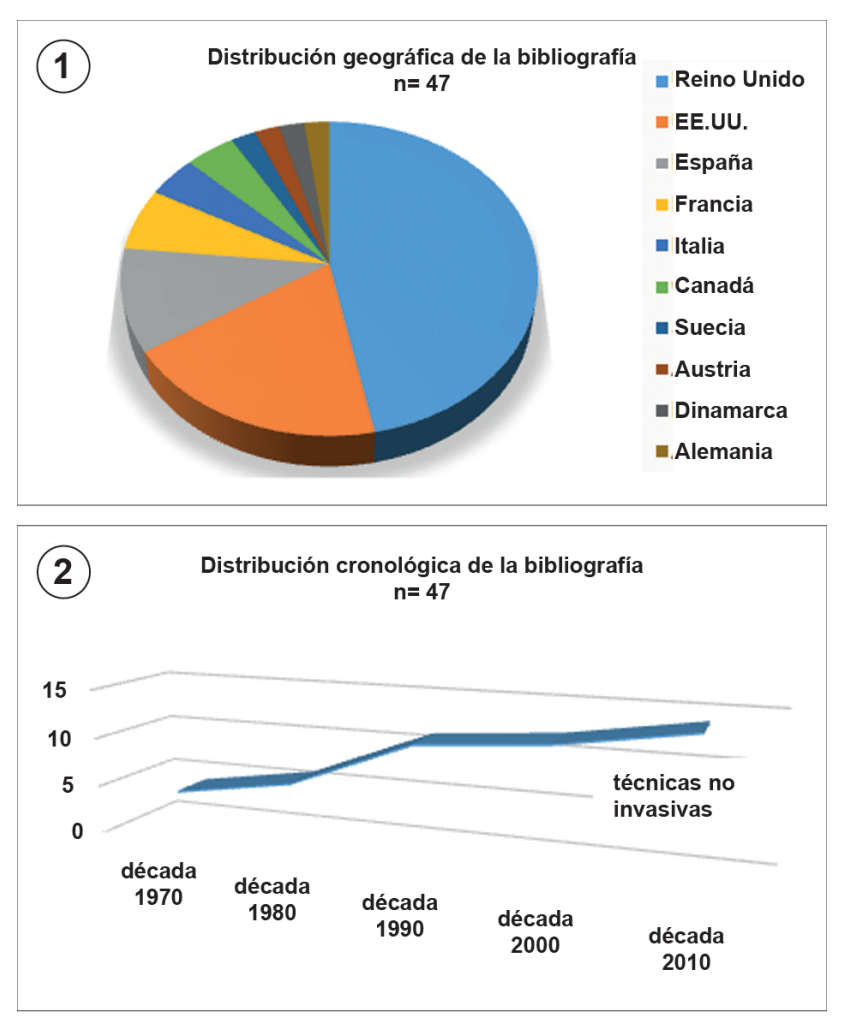

(3) Distribución geográfica de los autores más citados
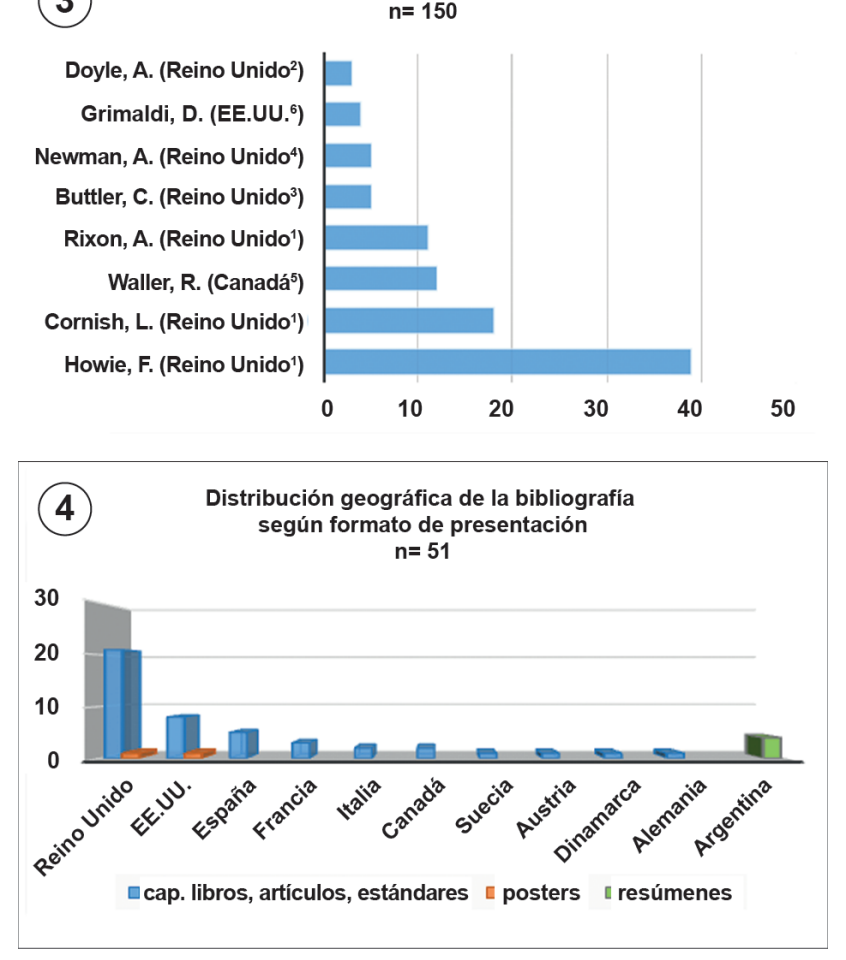

\section{CONCLUSIONES}

Las colecciones paleontológicas pueden deteriorarse por factores ambientales inapropiados. Las recomendaciones generales indican evitar las fluctuaciones de HR y temperatura, usar contenedores para evitar el polvo y los agentes biológicos, mantener los espacios limpios y proteger los ejemplares de los rayos UV, teniendo en cuenta que el daño es acumulativo.

Desde hace décadas y a nivel mundial, se está asistiendo a un cambio cultural en el cuidado y el manejo de las colecciones paleontológicas. La conservación preventiva que en sus inicios fue poco valorada, parece consolidarse con el análisis de casos de estudio, la utilización de técnicas no invasivas y la publicación de más artículos. En Argentina, sin embargo, la conservación preventiva como herramienta para la protección del patrimonio no ha tenido igual reconocimiento en el ámbito de las ciencias naturales en general y particularmente en la paleontología. Desde los primeros aportes de Francisco Muñiz y luego paulatinamente a partir de los estudios de fines del siglo XIX y hasta la fecha, la paleontología es reconocida como un área de alto desarrollo en las geociencias; las técnicas de preparación y de laboratorio acompañaron este proceso. Sin embargo, el abordaje en el campo de la conservación preventiva es apenas incipiente en nuestro país.

La identificación de las causas de esta realidad excede el tratamiento de este trabajo, pero no se descarta que estén motivadas por la falta de apoyo institucional y de recursos financieros para las colecciones que forman parte del patrimonio de museos, universidades y organismos gubernamentales, por la ausencia de información y capacitación, o quizá por la consideración de que el manejo y la conservación de ejemplares paleontológicos son temas menores. Esta contribución aboga por una concientización y valoración académica en el manejo y cuidado de las colecciones paleontológicas en la República Argentina.

Figura 2. Análisis de la bibliografía revisada. 1, Distribución geográfica de la bibliografía. 2, Distribución cronológica de la bibliografía. 3, Distribución geográfica de los autores más citados. 4, Distribución geográfica de la bibliografía según formato de presentación. Referencias de afiliaciones institucionales de autores: ${ }^{B}$ British Museum (Natural History), Inglaterra, Reino Unido; ${ }^{2}$ Natural History Museum, Inglaterra, Reino Unido; ${ }^{3}$ Amgueddfa Cymru, Gales, Reino Unido; ${ }^{4}$ University of Newcastle, Inglaterra; Reino Unido; ${ }^{5}$ Canadian Museum of Nature, Canadá; ${ }^{6}$ American Museum of Natural History, Estados Unidos de América. 


\section{AGRADECIMIENTOS}

$\mathrm{Mi}$ agradecimiento a E. Cerdeño por la lectura del manuscrito original y sus valiosas sugerencias. Mi sincero reconocimiento al Consejo Nacional de Investigaciones Científicas y Técnicas de Argentina (CONICET) por la Beca Externa CPA 2016, a F. Marcos Fernández (Universidad Complutense de Madrid), al Museo Geominero (Madrid) y al Museo Nacional de Ciencias Naturales (Madrid). Agradezco además a M. Paris, S. Álvarez, J. D’Angelo, A. Luy y N. Mónaco por sus respectivas contribuciones. Los comentarios de los tres revisores anónimos y del Equipo de la PE-APA enriquecieron este trabajo.

\section{REFERENCIAS}

Allington-Jones, L. y A. Trafford. (2017). The Airless Project. Evolving Ideas: Provocative new ways of working with collections. Natural Sciences Collections Association Conference. Cambridge. http://www.natsca.org/poster/2384

Álvarez, S. M. (2012). Recientes avances en las Colecciones de Vertebrados fósiles del Museo Argentino de Ciencias Naturales "Bernardino Rivadavia". Ameghiniana, Suplemento Resúmenes, 49(4), 84R

Andrew, K. J. (1996). A summary of the care and preventive conservation of sub-fossil bone for the non-specialist or Pleistocene problems- the sub-fossil bone scenario. The Biology Curator, 5, 24-28.

Baars, C. y Horak, J. (2018). Storage and conservation of geological collections-a research agenda. Journal of the Institute of Conservation, 41(2), 154-168. https://doi.org/10.1080/19455224. 2018.1463921

Baeza Chico, E. y Menéndez, S. (2005). Conservación y Restauración de ammonites piritizados del Museo Geominero (IGME, Madrid). $2{ }^{\circ}$ Congreso del Grupo Español del International Institute for Conservation of historic and artistic work (p. 47). Barcelona.

Barrón-Ortiz, C. I., Sawchuk, M. R., Li, C. y Jass, C. N. (2018). Conservation of subfossil bones from a lacustrine setting: Uncontrolled and controlled drying of Late Quaternary vertebrate remains from Cold Lake, Western Canada. Collection Forum, 32(1-2), 113. https://doi.org/10.14351/0831-4985-32.1.1

Bassett, M. G. (1979). Curation of palaeontological collections. Special Papers in Palaeontology, 22, 1-280.

Bather, F. A. (1908). The preparation and preservation of fossils. The Museums Journal, 8, 76-91.

Becherini, F., Del Favero, L., Fornasiero, M., Guastoni, A. y Bernardi, A. (2018). Pyrite decay of large fossils: The case study of the Hall of Palms in Padova, Italy. Minerals, 8(2), 40. https://doi.org/ 10.3390/min8020040

Bisulca, C., Nascimbene, P. C., Elkin, L. y Grimaldi, D. A. (2012). Variation in the deterioration of fossil resins and implications for the conservation of fossils in amber. American Museum Novitates, 3734, 1-19. https://doi.org/10.1206/3734.2

Buttler, C. J. (1994a). Environmental effects on geological material: Pyrite decay. En R. E. Child (Ed.), Conservation of Geological Collections (pp. 4-8). Archetype Publications Limited.

Buttler, C. J. (1994b). Damage to sub-fossil bone. En R. E. Child (Ed.), Conservation of Geological Collections (pp. 9-13). Archetype Publications Limited.

Collinson, M. E. (1986). Special problems in the conservation of palaeobotanical material. Geological Curator, 4(7), 439-445.

Cornish, L. (1986). The treatment of decaying pyritiperous fossil material using ethanolamine thioglycolate. Geological Curator, 4(7), 451-454.

Cornish, L. y Doyle, M. A. (1984). Use of ethanolamine thioglycolate in the conservation of pyritized fossils. Palaeontology, 27(2), 421-424.

Corral, J. C. (1999). La conservación del ámbar: revisión de los principales agentes de deterioro y soluciones publicados. Estudios del Museo de Ciencias Naturales de Álava, 14(2), 23-32.

Corral, J. C. (2012). Técnicas aplicadas en la preparación de un cráneo cuaternario de Panthera pardus (Linneo, 1758) de Ataun (cueva Allekoaitze, Guipúzcoa, España). Boletín Geológico y Minero, 123(2), 127-138.

Crespo, C. y Viñas, V. (1984). La preservación y restauración de documentos y libros en papel: un estudio del RAMP con directrices. Programa General de Información y UNISIST, Organización de las Naciones Unidas para la Educación, la Ciencia y la Cultura. http://unesdoc.unesco.org/images/0006/000635/063519so.pdf

de Guichen, G. (1999). La conservación preventiva: ¿Simple moda pasajera o cambio trascendental? Museum International, 201(51), 4-6.

Del Favero, L., Fornasiero, M., Molin, G., Reggiani, P. y Zorzi, F. (2012). II restauro dei vegetali fossili esposti nella "Sala delle Palme" del Museo di Geologia e Paleontologia dell'Università di Padova. Museologia Scientifica, 6(1-2), 49-57.

Doyle, A. M. (1983). The conservation of sub-fossil bird bone. Geological Curator, 3(7), 447-451.

Doyle, A. M. (2003). A large scale "Microclimate" enclosure for pyritic specimens. Geological Curator, 7(9), 329-335.

Druetta, S., Salas, M. J. y Sterren, A. F. (2017). Acciones de conservación preventiva en la "Colección Centro de Investigaciones Paleobiológicas" (CIPAL). Ameghiniana, Suplemento Resúmenes, 54(4), 77R.

Elder, A., Madsen, S., Brown, G., Herbel, C., Collins, C., Whelan, S., Wenz, C., Alderson, S. y Kronthal, L. (1997). Adhesives and consolidants in geological and paleontological applications: A Wall Chart. SPNHC Leaflets, 1(2), 1-2.

Garat, G. y Milani, M. (2011). Técnicas de restauración de fósiles. Caso: húmero holotipo de Panamericansaurus schroederi Calvo y Porfiri, 2010. Ameghiniana, Suplemento Resúmenes, 48(4), 166R.

García Fernández, I. M. (2013). Historia de la conservación preventiva. Parte I. Geoconservación, 5, 27-41. https://doi.org/10.37558/ gec.v5i0.195

Grimaldi, D. (1993). The care and study of fossiliferous amber. Curator The Museum Journal, 36(1), 31-49. https://doi.org/10.1111/j. 2151-6952.1993.tb00775.x

Hodgkinson, E. S. y Martin, S. (2004). History and mineralisation of highly degraded pyrite fossil collection. [Informe $n^{\circ}$ IR/04/037]. British Geological Survey. http://nora.nerc.ac.uk/id/eprint/ 509280/1/IR04037.pdf

Holmberg, I. C. (2000). Different degradation effects on Miocene whale skeletal remains from Gram, Denmark, caused by the clay matrix. Collection Forum, 14(1-2). https://spnhc.org/wp-content/uploads/2018/11/CFAbs_2000_V14N12.pdf

Howie, F. M. P. (1978). Storage environment and the conservation of geological material. The Conservator, 2(1), 13-19.

Howie, F. M. P. (1979a). Museum climatology and the conservation of palaeontological material. Special Papers in Palaeontology, 22, 103-126.

Howie, F. M. P. (1979b). Physical conservation of fossils in existing collections. Newsletter of the Geological Curators Group, 2(5), 269-280.

Larkin, N. (2011). Pyrite Decay: cause and effect, prevention and cure. NatSCA News, 21, 35-43.

Luna, C. A., Prieto, M. C., Miño-Boilini, A. R., Espíndola, V. E. y Zurita, A. E. (2019). Conservación preventiva en la colección de verte- 
brados fósiles de la Universidad Nacional del Nordeste, Argentina. Publicación Electrónica de la Asociación Paleontológica Argentina, 19(1R), R37-38.

Marcos-Fernández, F., Plaza Beltrán, M. y Ortega, F. (2018). La conservación preventiva de material paleontológico, la colección de Lo Hueco. $6^{\circ}$ Congreso del Grupo Español del International Institute for Conservation of historic and artistic work (pp. 402-410). Vitoria-Gasteiz.

McDonald, G., Elder, A. y Shelton, S. (2005). Curatorial Care of Paleontological and Geological Collections. En The Museum Handbook, Part l: Museum Collections (pp. U1-U54). National Park Service.

Montero, A. y Diéguez, C. (2001). Colecta y conservación de fósiles. Enseñanza de las Ciencias de la Tierra, 9(2), 121-126.

Museums and Galleries Commission. (1992). Standards in the museum care of biological collections. Spin Offset Limited.

Museums, Libraries and Archives Council. (2004). Standards in the Museum Care of Geological Collections. https://collectionstrust.org.uk/ resource/geological-collections-a-guide-to-standards-in-theirpreservation-and-care/

National Park Service. (1998). Storage concerns for geological collections. Conserve O Gram, 11(2), 1-4.

Neuhaus, B., Schmid, T. y Riedel, J. (2017). Collection management and study of microscope slides: Storage, profiling, deterioration, restoration procedures, and general recommendations. Zootaxa, 4322(1), 1-173. https://doi.org/10.11646/zootaxa.4322.1.1

Newman, A. (1998). Pyrite oxidation and museum collections: a review of theory and conservation treatments. Geological Curator, 6(10), 363-371.

Oddy, A. (2011). Harold Plenderleith and the conservation of antiquities and works of art. Intervención (México DF), 2(4), 56-62.

Odin, G. P., Chabard, D. y Rouchon, V. (2013). Étude de collections de paléontologie. De la caractérisation à la reproduction des altérations de fossiles dits pyriteux. En CeROArt (Online), Hors Série. http://journals.openedition.org/ceroart/3409

Pastorelli, G., Richter, J. y Shashoua, Y. (2011). Photoageing of Baltic amber - Influence of daylight radiation behind window glass on surface colour and chemistry. Polymer Degradation and Stability, 96(11), 1996-2001.

Rojas, A., Cabrera, F. y Corona, A. (2020). Temperature and relative humidity in a palaeontological collection: the buffering effect of microenvironments. Annales de Paléontologie, 106(4), 102446. https://doi.org/10.1016/j.annpal.2020.102446.
Rouchon, V., Badet, H., Belhadj, O., Bonnerot, O., Lavédrine, B., Michard, J.-G. y Miska, S. (2012). Raman and FTIR spectroscopy applied to the conservation report of paleontological collections: identification of Raman and FTIR signatures of several iron sulfate species such as ferrinatrite and sideronatrite. Journal of Raman Spectroscopy, 43(9), 1265-1274.

Shelton, S. Y. (1994). Conservation of vertebrate paleontological collections. En P. Leiggi y P. May (Eds.), Vertebrate paleontological techniques (vol. 1, pp. 3-34). Cambridge University Press.

Shinya, A. y Bergwall, L. (2007). Pyrite oxidation: Review and prevention practices. 67th Annual Meeting of the Society of Vertebrate Paleontology, Austin. https://vertpaleo.org/education/documents/Shinya_and_Bergwall_2007.pdf

Shute, C. H. y Cleal, C. J. (1987). Palaeobotany in Museums. Geological Curator, 4(9), 553-559.

Simmons J. E. y Muñoz-Saba, Y. (2005). Conservación preventiva y causa de deterioro de las colecciones. En J. E. Simons e Y. Muñoz-Zaba (Eds.), Cuidado, manejo y conservación de las colecciones biológicas: Conservación Internacional Serie Manuales para la conservación 1 (pp. 54-71). Universidad Nacional de Colombia.

Sterflinger, K. (2010). Fungi: Their role in deterioration of cultural heritage. Fungal Biology Review, 24(1-2), 47-55. https://doi.org/ 10.1016/j.fbr.2010.03.003

Stooshnov, A. y Buttler, C. (2001). The treatment of specimen labels affected by pyrite decay. Geological Curator, 7(5), 175-180.

Waddington, J. (2000). Conservation guidelines for invertebrate paleontology collections. The Paleontological Society Special Publications, 10, 93-104. https://doi.org/10.1017/S2475262200009011

White, R. D. (2000). Guidelines for the documentation and care of invertebrate fossil collections. The Paleontological Society Special Publications, 10, 51-64. https://doi.org/10.1017/S2475262200008960

doi: 10.5710/PEAPA.05.12.2020.341

Recibido: 23 de septiembre 2020

Aceptado: 5 de diciembre 2020

Publicado: 16 de junio 2021
Acceso Abierto Open Access

This work is licensed under

CC BY-NC 4.0

()(1) 